\title{
Collapsing chaos
}

\author{
Pabel Shahrear, Leon Glass, Roderick Edwards
}

\begin{abstract}
Genetic networks play a fundamental role in the regulation and control of the development and function of organisms. A simple model of gene networks assumes that a gene can be switched on or off as regulatory inputs to the gene cross critical thresholds. In studies of dynamics of such networks, we found unusual behavior in which phase plane trajectories display irregular dynamics that shrink over long times. This observation leads us to identify a type of dynamics that can be described as collapsing chaos, in which the Lyapunov exponent is positive, but points on the trajectory approach the origin in the long time limit.
\end{abstract}

Pabel Shahrear

Department of Mathematics, Shah Jalal University of Science and Technology, Sylhet - 3114, Bangladesh

e-mail: pabelshahreardyahoo.com

Leon Glass

Department of Physiology, 3655 Promenade Sir William Osler, McGill University, Montreal, Quebec, Canada H3G1Y6

e-mail: leon.glass@mcgill.ca

Roderick Edwards

Department of Mathematics and Statistics, University of Victoria, P.O. Box 1700 STN CSC, Victoria, B.C. Canada

e-mail:edwardseuvic.ca

Citation: Pabel Shahrear, Leon Glass, Roderick Edwards, Collapsing chaos, in R. Anguelov, M. Lachowicz (Editors), Mathematical Methods and Models in Biosciences, Biomath Forum, Sofia, 2018, pp. 35-43, http://dx.doi.org/10.11145/texts.2017.12.163

Copyright: (C) 2018 Shahrear et al. This article is distributed under the terms of the Creative Commons Attribution License (CC BY 4.0) which permits unrestricted use, distribution, and reproduction in any medium, provided the original author and source are credited. 


\section{Introduction}

One of the idealizations of genetic regulatory networks is that gene expression is regulated in a combinatorial fashion. Each gene is switched "on" or "off" by some combination of protein transcription factors. Modern research in genetic control has focused on the network wiring diagrams for regulatory control [6]. In work going back to the 1970s [8, 9], our group has proposed a mathematical formulation that develops piecewise linear models for this type of regulatory control. In this work, transcription factors are both produced and destroyed continually. Each transcription factor has a threshold and as it passes the threshold, the production of other transcription factors to which it is an input are either turned on or off or there is no effect: what happens in each case depends on the particular control functions assumed. As discussed in prior work, these piecewise linear equations can exhibit a large number of different dynamical properties including stable steady states, stable limit cycles, chaotic dynamics, and multiple attractors [4, 5]. A variety of modifications of the original equations is possible to include the effects of continuous rather than switching control, ot to include multiple thresholds for the control.

We have been working to develop techniques to predict possible nonlinear dynamical behaviors of a given network based on the wiring diagram of the network. One strategy we have used is to generate random networks and then analyze the resulting dynamics. In a recent paper we described weird dynamic behavior in a four dimensional network [13]. In the following, we focus attention on one unusual aspect of the dynamics observed in this example: an apparently shrinking or collapsing chaotic attractor.

In Section 2, we present a simple negative feedback system to introduce the class of models that we study. In Section 3, we briefly review the Lyapunov exponent and its relevance to the study of sensitive dependence on initial conditions. We also give a simple example that demonstrates that the Lyapunov exponent alone is not adequate to understand the long time dynamics in a simple system. Finally, in Section 4 we present numerical evidence of a more complicated system that has interesting long term dynamics that we term "collapsing" chaos.

\section{A simple negative feedback system}

In a negative feedback system, the ouput of a system acts to inhibit one of the steps of its production. The simplest type of negative feedback system would have two variables: say $y_{1}$ and $y_{2}$ where $y_{1}$ activates or stimulates the production of $y_{2}$ and $y_{2}$ inhibits the production of $y_{1}$. One representation of such a system is [7] 


$$
\begin{aligned}
& \frac{d y_{1}}{d t}=\frac{\lambda_{1} \theta_{1}^{n}}{y_{2}^{n}+\theta_{1}^{n}}-\gamma_{1} y_{1}, \\
& \frac{d y_{2}}{d t}=\frac{\lambda_{2} y_{1}^{n}}{y_{1}^{n}+\theta_{2}^{n}}-\gamma_{2} y_{2},
\end{aligned}
$$

where $\lambda_{i}, \gamma_{i}$ and $\theta_{i}$ represent production constants, decay constants, and thresholds, respectively. To make matters simple, we assume that $\lambda_{i}=1, \gamma_{i}=1$, and $\theta_{i}=0.5$, for $i=1,2$. In this model system, the control of production is governed by the Hill function

$$
h(y)=\frac{y^{n}}{y^{n}+\theta^{n}},
$$

where $\theta=\theta_{1}=\theta_{2}=0.5$.

In Figure 1, we show the dynamics from numerical integration assuming $n=4$ in panel (a) and $n=40$ in panel (b). There is a decaying, oscillatory approach to the fixed point at $(0.5,0.5)$. Due to the high value of the Hill coefficient $n$ in panel (b), the trajectories are approximated by straight lines suggesting the following strategy for analysis.

a)

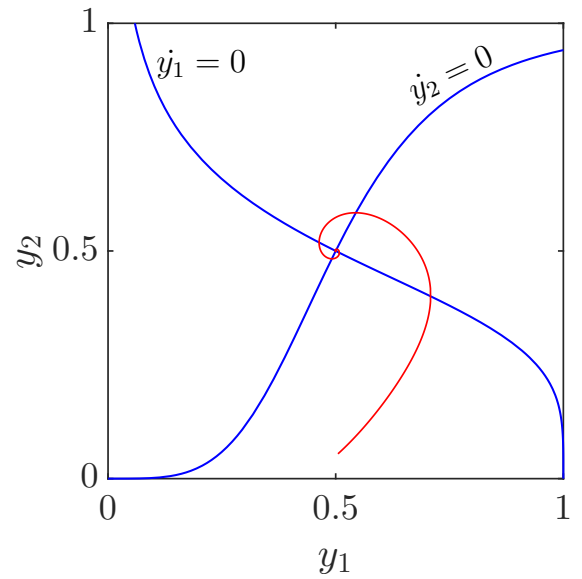

b)

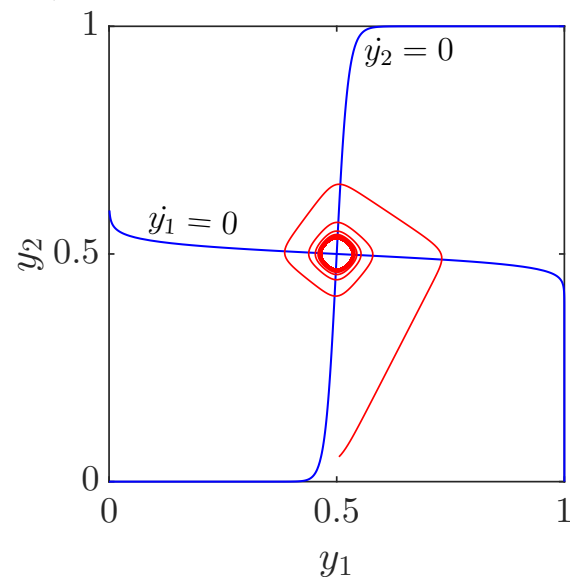

Fig. 1 Equation $\Omega_{1}$ in the $y_{1}-y_{2}$ plane. The trajectory is in red, and the isoclines are in blue, for $\lambda_{i}=1, \gamma_{i}=1$, and $\theta_{i}=0.5$, for $i=1,2$. (a) $n=4$; (b) $n=40$. There is an approach to the fixed point at $(0.5,0.5)$ in both cases. In the lower right "quadrant" of panel (b) the trajectory is approximated by a straight line directed towards the point $(1,1)$.

In the limit as $n \rightarrow \infty$ the sigmoidal Hill functions can be written in terms of the Heaviside function,

$$
\lim _{n \rightarrow \infty} h(y)=H\left(y-\theta_{i}\right),
$$

where $H(u)=1$ for $u \geq 0$ and $H(u)=0$ for $u<0$. 
For convenience and following our early analysis [9], we shift the fixed point to the origin by defining $x_{i}=2\left(y_{i}-\theta_{i}\right)$. Defining $X_{i}=H\left(\frac{x_{i}}{2}\right)$, in the new coordinate system in the limit $n \rightarrow \infty$, the dynamics are given by

$$
\begin{aligned}
& \frac{d x_{1}}{d t}=-1+2 \bar{X}_{2}-x_{1}, \\
& \frac{d x_{2}}{d t}=-1+2 X_{1}-x_{2},
\end{aligned}
$$

where the overbar indicates negation, $\bar{X}_{i}=1-X_{i}$. This is a piecewise linear system whose dynamics can be determined by simple construction. There are 4 focal points at $(1,1),(1,-1),(-1,-1),(1,-1)$. The flow in each quadrant is a straight line directed towards the focal point in the next quadrant in a counterclockwise direction. Thus, for an initial condition $\left.x_{1}(0) \geq 0, x_{2}(0)<0\right)$ by direct integration the trajectory is given by

$$
\begin{aligned}
& x_{1}(t)=1+\left(x_{1}(0)-1\right) e^{-t} \\
& x_{2}(t)=1+\left(x_{2}(0)-1\right) e^{-t}
\end{aligned}
$$

or

$$
\frac{x_{1}(t)-1}{x_{1}(0)-1}=\frac{x_{2}(t)-1}{x_{2}(0)-1} .
$$

Starting at an initial condition of $\left(0,-a_{0}\right)$, Eq. 6 holds until $x_{2}(t)=0$. Calling $a_{1}$ the value of $x_{1}$ when $x_{2}(t)=0$, we find $a_{1}=a_{0} /\left(1+a_{0}\right)$. In similar fashion, defining $a_{n}$ the distance of the trajectory from the origin on the $n$th threshold crossing, we have

$$
a_{n+1}=\frac{a_{n}}{1+a_{n}} .
$$

By iterating we find that

$$
a_{n}=\frac{a_{0}}{1+n a_{0}}
$$

so that $\lim _{n \rightarrow \infty} a_{n}=0$. The behavior of the Eqs. (4) and (7) is exploited in the examples that follow.

\section{The dyadic map in a shrinking domain}

One of the hallmark features of chaotic dynamics is sensitive dependence on initial conditions. Sensitive dependence on initial conditions is typically characterized by the Lyapunov exponent, which is the natural logarithm of the leading eigenvalue. A positive Lyapunov exponent in a nonlinear system is often taken as one of the factors used to define chaos [1, 3]. However, there are a number of examples that illustrate that a positive Lyapunov exponent is not necessarily synonymous with sensitive dependence on initial conditions [2, 11], or with a positive Lyapunov exponent of a linearization of the system (the Perron effect) [12], or with existence of a complex 
attractor (even in a bounded system) [10]. In [10], the positive Lyapunov exponent is transient, and is eventually lost. We provide an example in which the positive Lyapunov exponent is not lost, but nevertheless all trajectories asymptotically collapse to a fixed point.

Consider the following simple difference equation

$$
\begin{aligned}
& y_{n+1}=2 y_{n} \bmod a_{n} \\
& a_{n+1}=\frac{a_{n}}{1+a_{n}}
\end{aligned}
$$

with the initial condition of $a_{0}=1,0<y_{1}<1$.

Consider the sequence of values of $y_{n}$. In this example, the Lyapunov exponent is $\ln 2$. However, $\lim _{n \rightarrow \infty} y_{n}=0$. Thus, there is a positive Lyapunov exponent in a system that converges to 0 . We call this phenomenon "collapsing chaos".

\section{Four-dimensional gene network}

In Section 2, we discussed the dynamics in a continuous nonlinear and piecewise linear model of a negative feedback system. This system can also be taken as model genetic network. In previous work, we have examined the general class of models in which we similarly have piecewise linear equations [4, 5, 13]. The following set of equations was found in studies of randomly generated 4-dimensional piecewise linear models of gene networks [13].

$$
\begin{aligned}
\frac{d x_{1}}{d t} & =2 \bar{X}_{4}-1-x_{1} \\
\frac{d x_{2}}{d t} & =2\left(\bar{X}_{3} X_{1}+X_{1} X_{3} X_{4}+\bar{X}_{1} \bar{X}_{3} X_{4}\right)-1-x_{2} \\
\frac{d x_{3}}{d t} & =2\left(\bar{X}_{1} \bar{X}_{4}+X_{1} X_{2}\right)-1-x_{3} \\
\frac{d x_{4}}{d t} & =2 X_{1}-1-x_{4}
\end{aligned}
$$

where $x_{i}$ are continuous variables, $X_{i}$ are logical variables defined by $X_{i}=H\left(x_{i}\right)$, where $H(x)$ is the Heaviside step function, $\bar{X}_{i}=1-X_{i}$ for each $i=1, \ldots, 4$.

There are several different ways to visualize the dynamics in this system. In Figure 2. we show projections in the subspaces defined by taking each pair of two variables following a transient of 9000 threshold crossings. This projection shows that in the $x_{1} x_{4}$ subspace there is a decaying oscillation, reflecting the negative feedback loop between $x_{1}$ and $x_{4}$ in which $x_{1}$ activates $x_{4}$ and $x_{4}$ inhibits $x_{1}$. This decaying oscillation, in turn, acts as a decaying periodic input to $x_{2} x_{3}$. The projection on the $x_{2} x_{3}$ plane has a weird geometry with spike and cogwheel projections. As we analyzed in our previous paper [13], the interaction between $x_{2}$ and $x_{3}$ is predominantly 

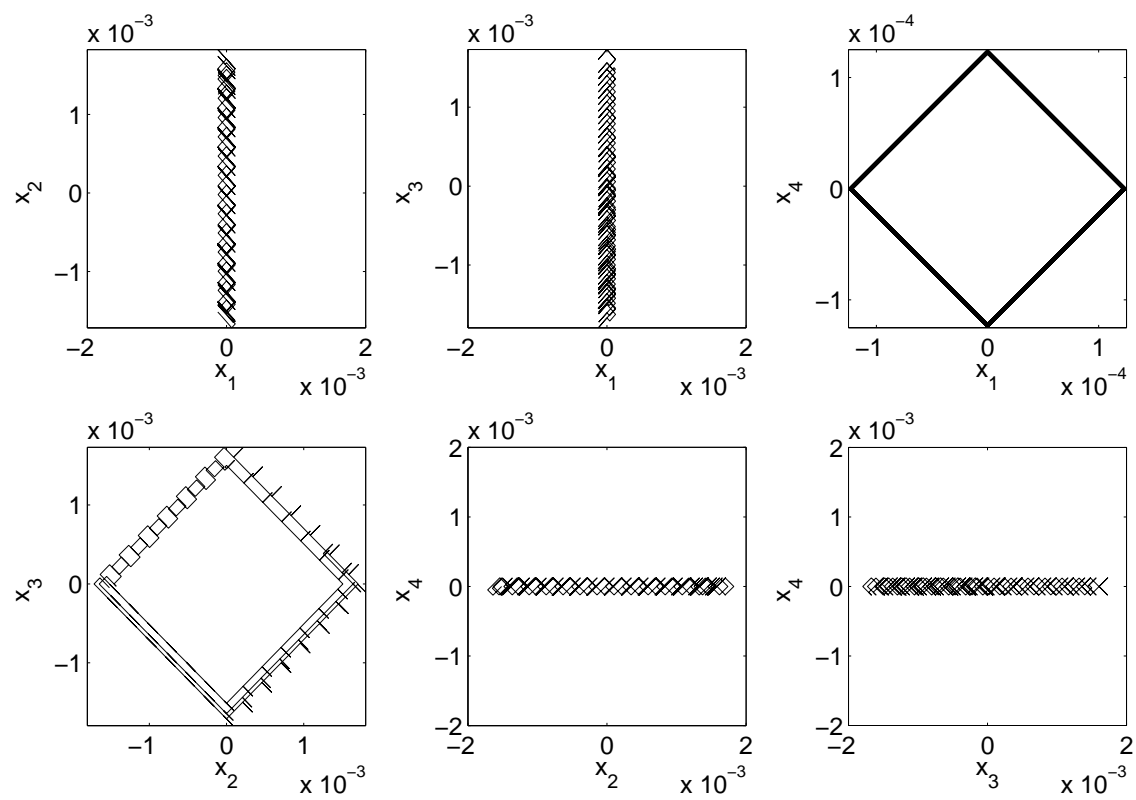

Fig. 2 Projections of Eq. 9 in the 6 subspaces defined by taking each pair of two variables. The projections are plotted for 300 threshold crossing following a transient of 9000 threshold crossings from an initial condition of: $x_{1}=0.500, x_{2}=0.036, x_{3}=-0.057, x_{4}=0.500$. Reproduced from [13].

a negative feedback system also in which $x_{2}$ activates $x_{3}$ and $x_{3}$ inhibits $x_{2}$, but some of the states of this interaction are modulated by the inputs from $x_{1}$ and $x_{4}$.

Figure 3 shows the trajectories at two different times. As time progresses the amplitude of the oscillation decreases. This decrease can be demonstrated numericallly by plotting the amplitude (defined as the minimum value of $\left|x_{2}\right|+\left|x_{3}\right|$ for 3 consecutive threshold crossings) of the oscillation as a function of time, Figure 4 From the numerical simulation the amplitude does not decrease monotonically, but over the long term the ampltude is decreasing.

In order to analyze this model, we considered a modification of the system in which the amplitude and the period of the $x_{1} x_{4}$ oscillation was fixed. In this modified system, the trajectory is a square in the $x_{1} x_{4}$ plane with a constant amplitude $\left|x_{1}\right|+$ $\left|x_{4}\right|=m$. In [13], we assumed $m=1$, but any other value could have been taken. Due to the piecewise linear nature of the underlying equations, we were able to compute trajectories in the $x_{2} x_{3}$ plane. In this computation, the jumps in amplitude are scaled by $m$. However, matrices that govern the expansion and contraction (see Table VII in [13]) can be shown to be independent of $m$, and thus their eigenvalues are independent of $m$. Since all trajectories (apart from a set of measure 0) have a 

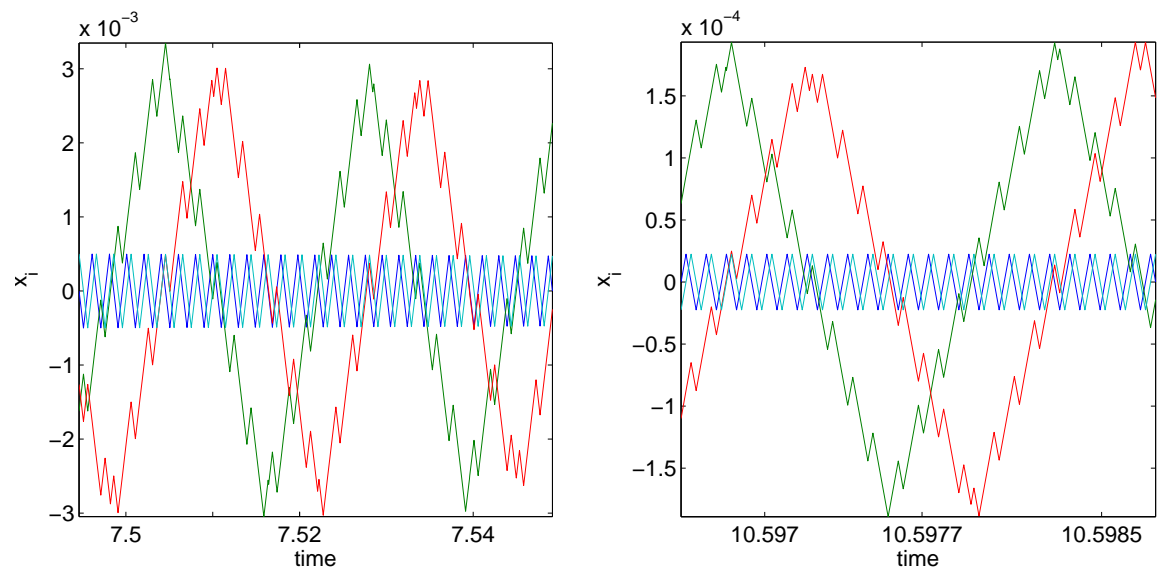

Fig. 3 Representative traces of the dynamics in Eq. 9 at two different times. Notice the difference in the scales. $x_{1}$ (dark blue), $x_{2}$ (green), $x_{3}$ (red), $x_{4}$ (light blue). Reproduced from [13].
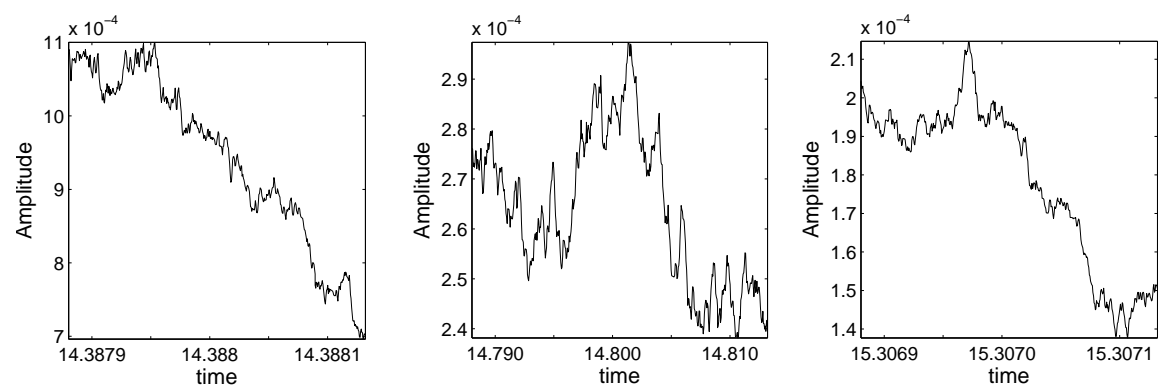

Fig. 4 Fluctuations of the amplitude in Eq.9 Reproduced from [13].

corresponding matrix with an eigenvalue $\lambda_{1}>1$, the maximal Lyapunov exponent is $\log \left(\lambda_{1}\right)>0$.

If we return to Eqs. (9), which include decay terms, the driving oscillator $\left(x_{1}, x_{4}\right)$ is damped and its amplitude converges to zero, so effectively, $m$ decreases and the size of the jumps in amplitude for the driven oscillator $\left(x_{2}, x_{3}\right)$ shrink. Furthermore, the decay terms for the driven oscillator apparently cause it too, to be damped though the random jumps are superimposed. We also expect that the independence of the Lyapunov exponents with respect to the jump size, $m$, for the non-damped version of the model also holds for the damped model. Thus, we conjecture that the entire system of Eq. (9) must converge asymptotically to the origin and displays collapsing chaos. 


\section{Conclusion}

We have shown that a particular 4-dimensional differential equation has positive Lyapunov exponents, producing irregular motion, which nevertheless collapses to the origin asymptotically. While it is much more complicated than the shrinking dyadic map of Section 3, the decay is quite similar in character in the two cases. We propose a concept of "collapsing chaos", since technically, a system in which the origin is globally asymptotically stable would not be considered chaotic (there is no chaotic attractor), but even here, positive Lyapunov exponents can cause mixing that appears chaotic locally in time.

\section{Acknowledgment}

Leon Glass thanks R. Anguelov for hospitality during the Biomath 2017 Conference in Skukuza Camp in Kruger National Park, South Africa. LG and RE have been supported by research grants from the Natural Sciences and Engineering Research Council, Canada. PS has been supported by the research grant PS/2017/29 from SUST Research Centre, Shahjalal University of Science and Technology, Sylhet 3114, Bangladesh. We thank Lucas Campanari for technical assistance.

\section{References}

1. Kathleen T. Alligood, Tim D. Sauer, and James A. Yorke. Chaos. Springer, 1996.

2. Bünyamin Demır and Şahin Koçak. A note on positive Lyapunov exponent and sensitive dependence on initial conditions. Chaos, Solitons \& Fractals, 12(11):2119-2121, 2001.

3. Jean-Pierre Eckmann and David Ruelle. Ergodic theory of chaos and strange attractors. Reviews of Modern Physics, 57(3):617, 1985.

4. Roderick Edwards. Analysis of continuous-time switching networks. Physica D: Nonlinear Phenomena, 146(1):165-199, 2000.

5. Roderick Edwards and Leon Glass. A calculus for relating the dynamics and structure of complex biological networks. Adventures in Chemical Physics, 132:151-78, 2005.

6. Mark B. Gerstein, Anshul Kundaje, Manoj Hariharan, Stephen G. Landt, Koon-Kiu Yan, Chao Cheng, Xinmeng Jasmine Mu, Ekta Khurana, Joel Rozowsky, Roger Alexander, et al. Architecture of the human regulatory network derived from ENCODE data. Nature, 489(7414):91, 2012.

7. Leon Glass. Combinatorial and topological methods in nonlinear chemical kinetics. Journal of Chemical Physics, 63(4):1325-1335, 1975.

8. Leon Glass and Stuart A. Kauffman. The logical analysis of continuous, non-linear biochemical control networks. Journal of Theoretical Biology, 39(1):103-29, 1973.

9. Leon Glass and Joel S. Pasternack. Stable oscillations in mathematical models of biological control systems. Journal of Mathematical Biology, 6(3):207-223, 1978.

10. Celso Grebogi, Edward Ott, and James A. Yorke. Crises, sudden changes in chaotic attractors, and transient chaos. Physica D: Nonlinear Phenomena, 7(1-3):181-200, 1983.

11. Hüseyin Koçak and Kenneth J. Palmer. Lyapunov exponents and sensitive dependence. Journal of Dynamics and Differential Equations, 22(3):381-398, 2010. 
12. Gennady A. Leonov and Nicolay V. Kuznetsov. Time-varying linearization and the Perron effects. International Journal of Bifurcation and Chaos, 17(4):1079-1107, 2007.

13. Pabel Shahrear, Leon Glass, and Rod Edwards. Chaotic dynamics and diffusion in a piecewise linear equation. Chaos: An Interdisciplinary Journal of Nonlinear Science, 25(3):033103, 2015. 